\title{
The X-ray properties of magnetic massive stars
}

\author{
Yaël Nazé ${ }^{1}$, Véronique Petit ${ }^{2}$, Melanie Rinbrand ${ }^{2}$, David Cohen ${ }^{3}$, \\ Stan Owocki ${ }^{2}$, Asif ud-Doula ${ }^{4}$ and Gregg Wade ${ }^{5}$ \\ ${ }^{1}$ FNRS/ULg, Dept AGO, Allée du 6 Août 17, B5C, 4000-Liège, Belgium \\ email: naze@astro.ulg.ac.be \\ ${ }^{2}$ Dept of Physics \& Astronomy, Univ. of Delaware, Bartol Res. Inst., Newark, DE 19716, USA \\ ${ }^{3}$ Dept of Physics \& Astronomy, Swarthmore College, Swarthmore, PA 19081, USA \\ ${ }^{4}$ Penn State Worthington Scranton, Dunmore, PA 18512, USA \\ ${ }^{5}$ Dept of Physics, RMC, PO Box 17000, Station Forces, Kingston, ON K7K 4B4, Canada
}

\begin{abstract}
Early-type stars are well-known to be sources of soft X-rays. However, this highenergy emission can be supplemented by bright and hard X-rays when magnetically confined winds are present. In an attempt to clarify the systematics of the observed X-ray properties of this phenomenon, a large series of Chandra and XMM observations was analyzed, over 100 exposures of $60 \%$ of the known magnetic massive stars listed recently by Petit et al. (2013). It is found that the X-ray luminosity is strongly correlated with mass-loss rate, in agreement with predictions of magnetically confined wind models, though the predictions of higher temperature are not always verified. We also investigated the behaviour of other X-ray properties (absorption, variability), yielding additional constraints on models. This work not only advances our knowledge of the X-ray emission of massive stars, but also suggests new observational and theoretical avenues to further explore magnetically confined winds.
\end{abstract}

Keywords. stars: early-type, stars: magnetic fields, X-rays: stars

\section{Introduction}

In the last decade, tens of massive stars were found to be strongly magnetic (e.g. Donati et al. 2002; Hubrig et al. 2011; Wade et al. 2012). Their overall properties appear quite similar to those of magnetic AB stars: the fields appear strong, stable, and organized on large scales (e.g. dipoles) but quite rare. Such magnetic fields are able to channel the stellar winds of massive stars towards the magnetic equator, giving rise to magnetically confined winds (MCWs). The shocks between the wind flows should give rise to an intense X-ray emission (Babel \& Montmerle 1997). Two MCWs prototypes, $\sigma$ Ori E and $\theta^{1}$ Ori $\mathrm{C}$, were readily found. Their high-energy emission appears in line with expectations (Gagné et al. 2005; Townsend et al. 2007), but the situation appears more confused for other objects (Oskinova et al. 2011), like for example the Of?p stars (Nazé et al. 2010): overluminosity and hard X-rays are not always the rule. As previous studies have focused on a limited number of objects, we have undertaken a systematic study of the full sample of magnetic massive stars, with the hope to derive the overall high-energy properties of MCWs (Nazé et al., submitted).

\section{X-ray data}

To ensure a high homogeneity as well as to maximize the number of detections, we focused on CCD spectra in the $0.5-10 . \mathrm{keV}$ range coming from XMM-EPIC and 

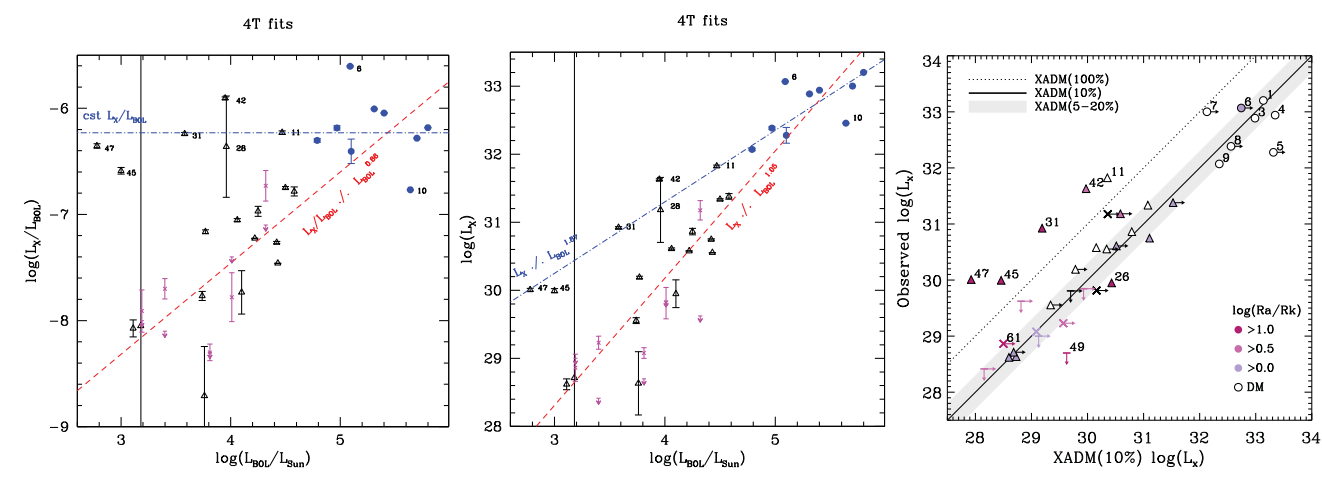

Figure 1. Left and middle: $\log \left(L_{\mathrm{X}} / L_{\mathrm{BOL}}\right)$ ratio and X-ray luminosity as a function of bolometric luminosity, along with best-fit relations. Right: Comparison of observed and predicted (using ud-Doula et al. models) luminosities.

Chandra-ACIS. High-resolution data are indeed few in number, ROSAT data do not sample the hard X-ray emission deemed to exist in such objects, ASCA has a poor resolution and cannot isolate the X-ray emission from our targets (which often lie in clusters), and Swift or Suzaku archives did not yield additional detections. Thus concentrating on Chandra and XMM data, we found that over a hundred exposures are available for 39 out of the 64 magnetic stars in the Petit et al. (2013) catalog. In the magnetic confinementrotation diagram (Petit et al. 2013), these targets are well distributed, so that our sample is not a particular subpopulation of the full catalog.

Amongst the objects covered by X-ray observations, there are 6 non-detections, 5 faint detections (only count rate available), and 28 detections bright enough for spectra to be extracted. These spectra were fitted by absorbed optically-thin plasma (with free temperatures or a set of fixed temperatures, both methods yielding similar results).

\section{X-ray luminosity}

If MCWs are responsible for producing X-rays, then some correlations between X-ray observables and stellar/wind/magnetic properties are expected. We first investigated the link between the X-ray emission levels and the mass-loss rates since shocks extract some of the wind kinetic energy to produce X-rays. Our sources can be separated in two groups (Fig. 1).

The first group comprises all O-stars and six B-stars which display $\log \left(L_{\mathrm{X}} / L_{\mathrm{BOL}}\right)=$ $-6.23 \pm 0.07$. This is equivalent to the relation $L_{\mathrm{X}} \propto \dot{M}^{0.6}$, which is typical of the $\mathrm{X}$-ray emission from O-stars and their radiative stellar winds. However, the magnetic objects display a significantly brighter X-ray emission than "normal" O-stars which have $\log \left(L_{\mathrm{X}} / L_{\mathrm{BOL}}\right) \sim-7$ (e.g. Nazé et al. 2011, and references therein). Amongst O-stars, note that there are two deviant cases, indicating a probable non-magnetic origin for the X-rays: $\zeta$ Ori, which has a very weak field hence a "normal", fainter X-ray emission, and Plaskett's star, whose brighter X-ray emission is probably linked to binary interactions rather than MCWs.

The second group, comprising most B-stars, displays $L_{\mathrm{X}} \propto \dot{M}^{1.4}$ or equivalently, since the winds are radiation-driven, $L_{\mathrm{X}} \propto \dot{L}_{\mathrm{BOL}}^{1.9}$ (though it may be noted that relations with $L_{\mathrm{BOL}}$ display a larger scatter than relations with $\left.\dot{M}\right)$. The trend is here much steeper, but it is still shallower than the $L_{\mathrm{X}} \propto \dot{M}^{2}$ relation for adiabatic shocks, adequate for embedded-wind shocks in low-density winds (Owocki et al. 2013). Indeed, wind confinement is likely to reduce significantly the amount of adiabatic cooling, since material in 
MCW shocks is trapped in closed loops and so does not undergo the adiabatic expansion cooling of an outflowing stellar wind.

The best-fit relations for O- and B-stars are shown in the right panel of Fig. 1. Note that faint/non-detections, though not included in the derivation of these best-fit relations, agree well with them.

We have examined alternative relations (with wind density or $\mathrm{H} \alpha$ emission strength) as well as more complicated relations (e.g. considering dependences on both $\dot{M}$ and magnetic field strength) but none was found significantly better.

Are these relations theoretically understood? Babel \& Montmerle (1997) proposed $L_{\mathrm{X}} \propto \dot{M} \times B^{0.4} \times v_{\infty}$ but this scaling seems to be inadequate for the lowest and highest mass-loss rates. Besides, the X-ray emission level predicted by Babel \& Montmerle formula is too high by 1.8 dex compared to observations (which probably comes from the nearly perfect conversion efficiency of that model). New MHD simulations by ud-Doula et al. (2014) re-investigated the problem of the X-ray emission of MCWs, demonstrating the impact of shock-retreat effects especially in low-density winds. Their predictions appear much better in line with the observational results, both for the emission levels and the observed trends with $\dot{M}$ (Fig. 1). A few discrepancies remain, though, which could not yet be explained. They concern the few B-stars with bright $L_{\mathrm{X}}$ but low $\dot{M}$ belonging to group 1. Their predicted luminosities are much lower than their observed ones. Unfortunately, we could not clearly identify a common feature amongst these objects. For example some of them are fast rotators but not all, and some supposed "twins" of these targets do not show a similar elevated X-ray emission.

\section{X-ray spectral shape}

Hardness ratios and average temperatures were also derived from the spectral fits. Strongly magnetic O-stars clearly are harder/hotter than their non-magnetic siblings, while most of the B-stars show low hardness ratios. However, no correlation was found with stellar/wind/magnetic parameters despite the fact that hotter plasma is theoretically expected for higher confinement values or higher mass-loss rates (ud-Doula et al. 2014). Also, the overluminous (compared to MHD predictions) B-stars do not necessarily stand up with particularly high or low temperatures. The drivers for X-ray luminosity and plasma temperature certainly appear different.

\section{X-ray absorption}

Spectral fits also allowed for the possibility of absorption in addition to the interstellar one, e.g. arising in the dense magnetospheres. In general, magnetic stars appear to behave as non-magnetic objects: an additional absorption is not needed for magnetic B-stars (as is usual for the "normal" ones), while O-stars require an amount comparable to what is needed for "normal" O-stars without MCWs. No correlation between that absorption and stellar/wind/magnetic parameters is found. The only special case is NGC1624-2, the most magnetic O-star, whose extremely dense magnetosphere leads to the presence of very high absorption (Petit et al., submitted). Again, as for temperatures, the overluminous B-stars do not show, as a group, particular values of the absorption. 

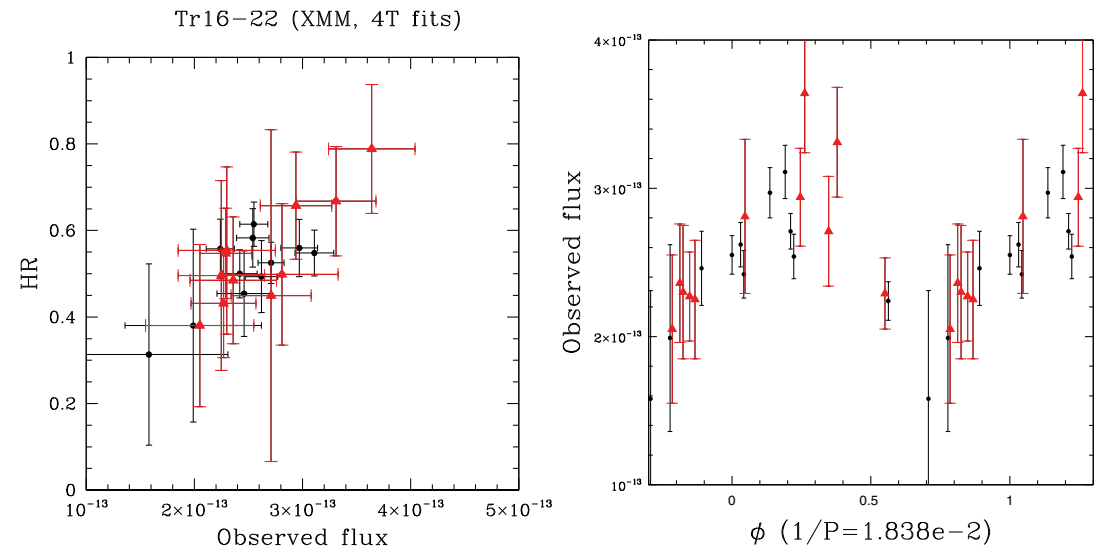

Figure 2. Variations of Tr16-22 in XMM data. Left: Correlation between flux and hardness changes. Right: Flux changes phased with the newly discovered 54d period. The 2003 data are shown in red.

\section{Variation of the X-ray emission}

When several exposures are available, we have examined the X-ray variability. Three behaviours are detected:

- Constant emission, as expected from the properties of the targets (pole-on geometry for HD148937, large magnetosphere for $\sigma$ Ori E, non-magnetic origin for the X-rays of $\zeta$ Ori).

- Flux changes without spectral changes (HD47777 and $\beta$ Cep), which cannot be linked to MCWs with current data. Note that these changes include the detection of flares in HD47777.

- Flux and spectral changes (Tr16-22, HD191612, NU Ori, $\theta^{1}$ Ori C), often found to be in harmony with the stellar rotation (HD191612, $\theta^{1}$ Ori $\mathrm{C}$ ). In this context, the large number of X-ray observations of Tr16-22 enabled us to identify its period, about 54d (Fig. 2, Nazé et al., submitted). The observed variations can be explained by occultation effects in stratified MCWs. Note however that two trends are seen: the X-ray emissions are either harder (Tr16-22, HD191612, NU Ori) or softer $\left(\theta^{1}\right.$ Ori C) when brighter. This suggests that the structure of MCWs may vary amongst magnetic objects, even if their stellar/wind/magnetic properties are apparently quite similar. Detailed models are now needed to explain this intriguing behaviour.

\section{Conclusion}

Using archival and dedicated XMM and Chandra observations, we have examined the X-ray behaviour of magnetic massive stars. More than half of the stars in Petit et al. catalog have been surveyed, and this sample is representative of the whole population.

We found that the X-ray luminosity is strongly correlated to the bolometric luminosity or, equivalently, to the mass-loss rate. However, two groups of objects can be defined: one with constant $\log \left(L_{\mathrm{X}} / L_{\mathrm{BOL}}\right) \sim-6.2$, and one with $L_{\mathrm{X}} \propto \dot{M}^{1.4}$. No alternative or more complex correlation was found significantly better. MHD models are able to reproduce the levels of X-ray emissions and the above relations for most objects - the exceptions are a few B-stars with an observed luminosity much brighter than expected on the basis of their mass-loss rate. No specific, common property could be identified for these outliers, whose origin remains unknown. 
The X-ray spectral shapes appear varied amongst targets, but no correlation with stellar, wind, or magnetic properties could be derived. In particular, the prediction of harder $\mathrm{X}$-rays for higher mass-loss or wind confinement is not verified, and the overluminous outliers do not stand up as peculiarly soft or hard X-ray sources. Similarly, the local absorptions do not appear to be correlated with stellar, wind, or magnetic properties.

Finally, we have examined the variability of the targets when several exposures were available. Constancy of the X-ray emission is found for some cases, and can be explained by the properties of the targets. At the other extreme, correlated flux and spectral changes are derived for some objects. These variations appear to be coherent with the stellar rotation period, hence can be explained by regular occultations of the X-ray emission regions by the stellar body. In that case, the presence of simultaneous spectral changes requires the MCWs to be stratified in temperature.

\section{References}

Babel, J. \& Montmerle, T. 1997, A\&A 323, 121

Donati, J.-F., Babel, J., Harries, T. J., et al. 2002, MNRAS 333, 55

Gagné, M., Oksala, M. E., Cohen, D. H., et al. 2005, ApJ 628, 986

Hubrig, S., Schöller, M., Kharchenko, N. V., et al. 2011, A\&A 528, A151

Nazé, Y., Broos, P. S., Oskinova, L., et al. 2011, ApJS 194, 7

Nazé, Y., Ud-Doula, A., Spano, M., et al. 2010, A\&\&A 520, A59

Oskinova, L. M., Todt, H., Ignace, R., et al. 2011, MNRAS 416, 1456

Owocki, S. P., Sundqvist, J. O., Cohen, D. H., \& Gayley, K. G. 2013, MNRAS 429, 3379

Petit, V., Owocki, S. P., Wade, G. A., et al. 2013, MNRAS 429, 398

Townsend, R. H. D., Owocki, S. P., \& Ud-Doula, A. 2007, MNRAS 382, 139

ud-Doula, A., Owocki, S., Townsend, R., Petit, V., \& Cohen, D. 2014, MNRAS 441, 3600

Wade, G. A., Grunhut, J., Gräfener, G., et al. 2012, MNRAS 419, 2459

\section{Discussion}

Alecian: Why do you think a Herbig Be star would flare?

NAZÉ: It may not be from the Herbig Be star itself - it is not uncommon for the X-ray emission of "normal" B stars to be considered as coming from (or contaminated by) that of a companion. If the star is an Herbig, then a companion would also be young, explaining the flaring activity. With the current data, it is not possible to firmly link the flares and the confined winds, as I said.

AERTS: Did you try to compare your X-ray data with EUVE data for the few pulsating B stars that now turn out to be magnetic, e.g., $\beta$ CMa (low amplitude non-radial pulsator), $\xi^{1}$ CMa (large amplitude radial pulsator)?

NAZÉ: We didn't do this comparison yet because EUVE data were not available for most of our targets, but I agree such a study would be valuable.

PULS: I just want to suggest (in future publications) that you call your "mass-loss rate" the "theoretical feeding rate", since the actual mass-loss rate is lower due to infall.

NAZÉ: Fully agree.

WeIs: You expect the X-ray emission to origin in the compression zone. If however this axis of rotation and the magnetic field are not aligned, this region is precessing and might be variable. 
NAZÉ: This is a possibility, yes, but which has not be explored (and cannot yet with the current data).

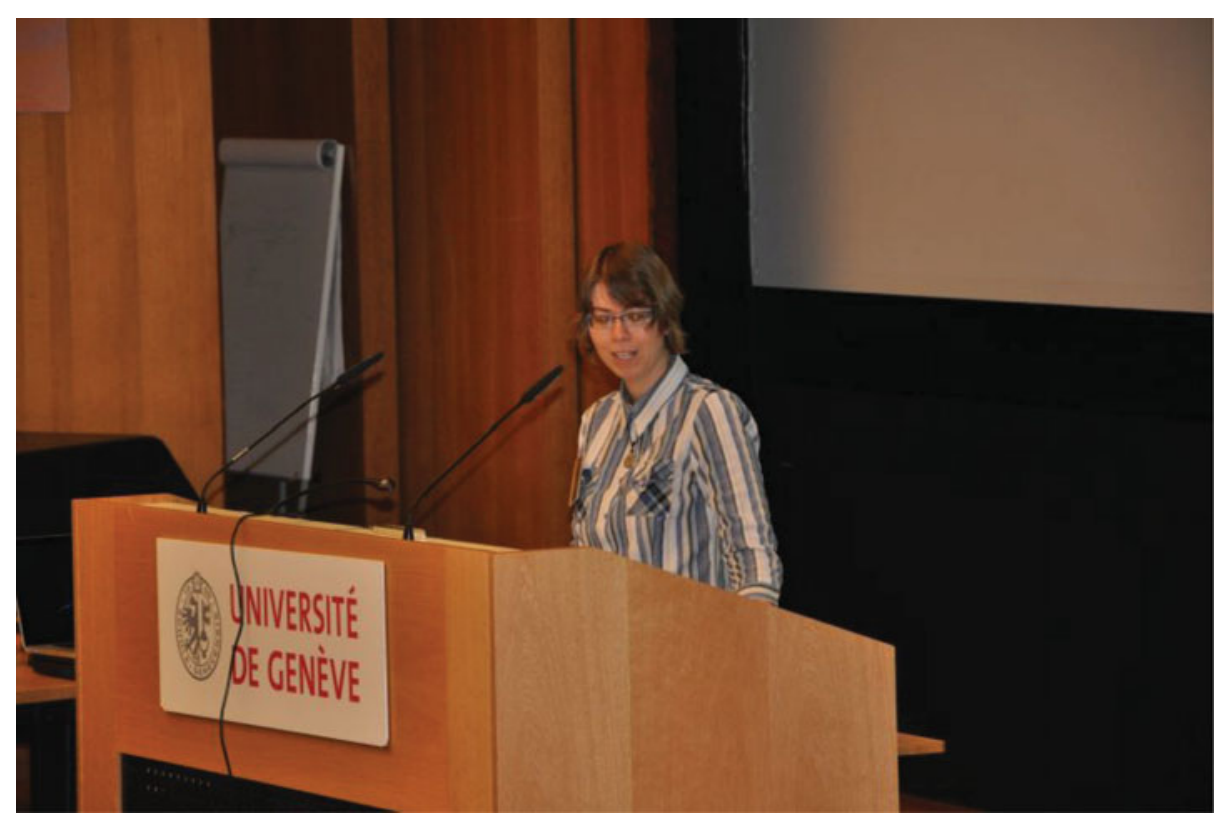

Yaël Nazé

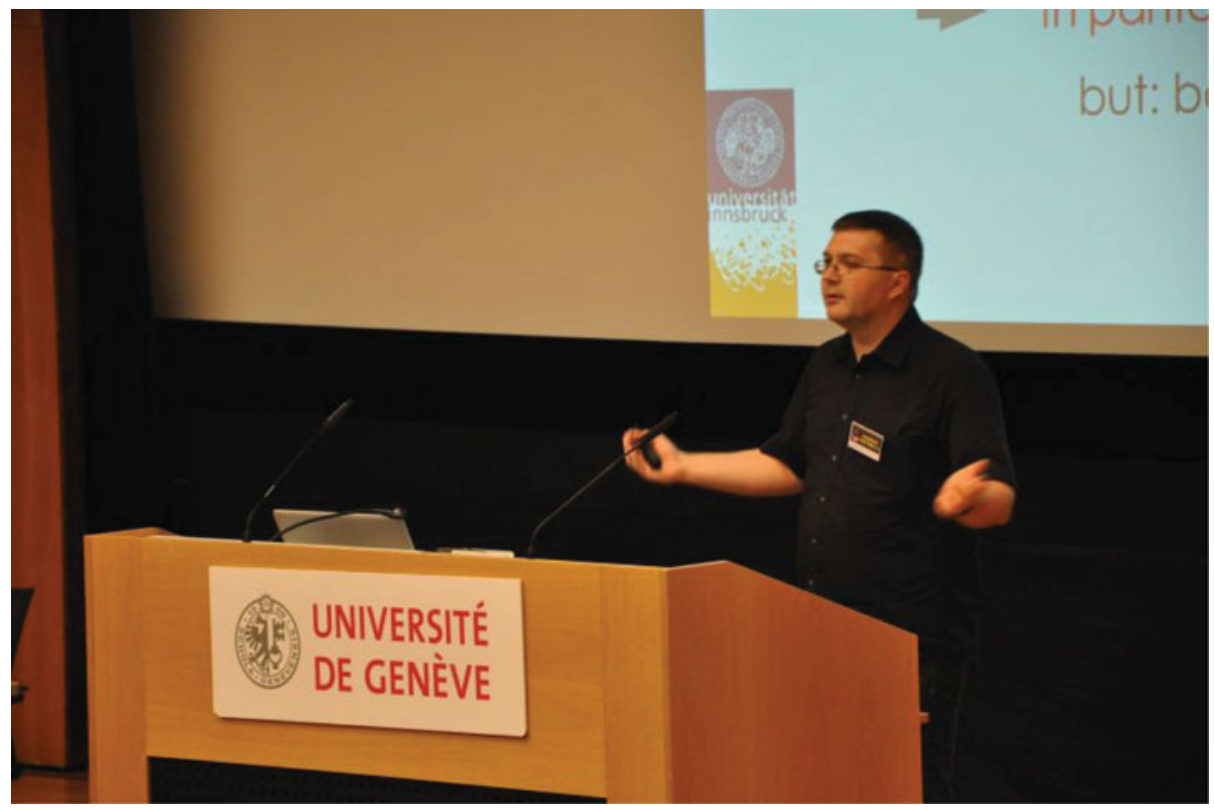

Norbert Przybilla 